\title{
Percepção dos acadêmicos de Odontologia em relação ao teste de progresso
}

\author{
Fabiana Aparecida Mayrink de Oliveira*; Marcelo Tarcísio Martins*, Antônio Marcio Lima Ferraz \\ Júnior*; Cleide Gisele Ribeiro**; Rodrigo Guerra de Oliveira***; Fernanda Ribeiro Porto* \\ * Mestre, Professor(a) da Faculdade de Odontologia da \\ FCMS/JF \\ ** Doutora, Professora da Faculdade de Odontologia da \\ FCMS/JF \\ *** Doutor, Coordenador da Faculdade de Odontologia da \\ FCMS/JF
}

Recebido em 30/12/2018. Aprovado em 02/05/2020.

\begin{abstract}
RESUMO
Este estudo teve como objetivo analisar a percepção dos acadêmicos de Odontologia em relação ao Teste de Progresso (TP). Após o término do TP, os 284 estudantes do curso receberam um questionário, que abordava a percepção do grau de dificuldade do teste na área básica e específica; a extensão da prova; clareza e objetividade dos enunciados; adequação das informações/instruções fornecidas para a resolução das questões; confirmação da abordagem dos conteúdos durante o curso; estudo dos conteúdos pelos estudantes e avaliação do tempo ofertado e utilizado pelos estudantes na resolução das questões. Empregou-se o coeficiente de correlação de Spearman e análise de correspondência. Desta forma, observou-se que o grau de dificuldade da prova na área básica e na área específica foi maior nos primeiros períodos e quanto à percepção do estudante em relação à extensão da prova, a maioria considerou adequada, bem como a clareza e objetividade do teste. Quanto à dificuldade na realização do teste, os primeiros períodos relataram o desconhecimento de todo o conteúdo e para os últimos períodos, houve falta de motivação. A maioria dos estudantes respondeu que utilizou entre uma e duas horas para realizar o teste. Conclui-se que a percepção dos acadêmicos demonstra que o TP está sendo realizado de forma adequada. Observou-se ainda que os períodos iniciais relataram desconhecimento do conteúdo, o que corrobora com o objetivo do teste, que é avaliar o ganho cognitivo dos estudantes à medida que avançam no curso.

Descritores: Avaliação. Taxonomia. Formação Profissional. Educação em Odontologia.
\end{abstract}




\section{INTRODUÇÃO}

O teste de progresso (TP) é um teste longitudinal, desenvolvido originalmente na década de 1970 pelas Universidades de Maastricht e Missouri, com a intenção de avaliar a evolução do desempenho cognitivo dos estudantes da graduação durante o curso e o próprio curso de maneira formativa ${ }^{1,2}$. Assim, o estudante tem a oportunidade de verificar seu desempenho nas diversas áreas do curso e do currículo, e ainda identificar suas fragilidades e fortalezas, para que através do processo de retroalimentação possa traçar seus planos de estudo e buscar a formação profissional $^{3}$.

No Brasil, o TP tornou-se cada vez mais popular nas escolas médicas, após a aplicação do primeiro teste na Universidade Estadual de Londrina (UEL). Atualmente, este teste vem sendo aplicado em várias escolas como na Universidade Estadual de Campinas (Unicamp), Universidade Federal de Santa Catarina (UFSC), Universidade Estadual Paulista (Unesp), Universidade Federal de São Paulo (Unifesp), Universidade de São Paulo (FMUSP e FMRP-USP), Universidade Regional de Blumenau (Furb) e Faculdade de Medicina de Marília (Famema) ${ }^{4}$.

Entretanto, apesar do TP ser bem estabelecido nas escolas médicas, na Odontologia ainda se encontra em estado de adaptação, pois mesmo com semelhanças no conhecimento básico, a precocidade da prática clínica no curso de Odontologia torna o aprendizado acentuadamente voltado ao ganho de habilidades práticas, o que pode interferir diretamente na forma de construir o teste $^{4}$. Assim, avaliações longitudinais pouco têm sido experimentadas em estudantes de graduação em Odontologia, com poucos exemplos nacionais como na Faculdade de Odontologia da USP e internacionais como no College of Medicine and Dentistry, no Reino Unido ${ }^{6}$. Percebe-se que a implementação do TP não é tão simples, pois envolve uma mudança de pensamento e também na cultura acadêmica de como realizar avaliações ${ }^{5,6}$.

Neste cenário, é importante considerar a avaliação do estudante com intuito de melhorar a forma de avaliar, assim como tem sido feito na Universidade do Porto em Portugal e na Universidade do Rio de Janeiro (UFRJ) ${ }^{7,8}$.

O propósito da avaliação da percepção dos estudantes é aperfeiçoar, recapitular ou exemplificar o processo de avaliação. Nos últimos anos, este tema tem sido constantemente lembrado na área da avaliação educacional devido à necessidade de os avaliadores melhorarem o seu trabalho, fundamentados em novos conceitos e instrumentos ${ }^{9}$. Embora a preocupação exista em diversos programas de autoavaliação das instituições de ensino superior, existe uma lacuna quanto à disseminação de relatórios e de referências teórico-metodológicas de como conduzi-la9.

A Faculdade de Ciências Médicas e da Saúde de Juiz de Fora (FCMS/JF) implementa o TP desde 2009 como instrumento de avaliação do curso de Odontologia. Portanto, o presente estudo objetivou analisar a percepção dos estudantes em relação ao TP do curso de Odontologia realizado em 2014.

\section{METODOLOGIA}

Esta pesquisa foi apreciada pelo Comitê de Ética em Pesquisa da FCMS/JF, que emitiu protocolo de aprovação $\mathrm{n}^{\circ} 697.155$.

O TP da Odontologia, realizado no primeiro semestre de 2014, contemplava 100 questões fechadas, com 5 distratores cada (representados pelas letras a, b, c, d e e) e abordou os seguintes conteúdos da área básica: Instrumentalização Linguística, Anatomia, Imunologia, Microbiologia, Biossegurança, Histologia / Odontogênese / Embriologia, Far-, macologia, Bioética e Saúde e Metodologia. Na área específica os conteúdos foram: Saúde coletiva/Epidemiologia, Patologia, Radiologia, Periodontia, Endodontia, Odontopediatria, 
Ortodontia, Oclusão, Disfunção Temporomandibular, Anestesiologia, Cirurgia, Dentística, Cariologia, Materiais Dentários e Prótese. Este foi aplicado aos 284 estudantes do curso, que compuseram a amostra deste estudo. A participação do acadêmico no TP é obrigatória na instituição.

Após o término do TP, o estudante recebeu um Termo de Consentimento Livre e Esclarecido que informava sobre os objetivos da avaliação, os riscos e benefícios do estudo e, assim que assinado, recebia um questionário de percepção do TP com oito questões com cinco distratores cada. O questionário aplicado foi baseado no questionário de percepção da prova do Exame Nacional do Desempenho dos Estudantes (Enade) do curso de Odontologia.

Foi exigida somente a identificação do período cursado pelo estudante e este respondeu às questões em anonimato. $\mathrm{O}$ conteúdo da avaliação abordou: a percepção do grau de dificuldade do teste na área básica (questão 1), a percepção do grau de dificuldade do teste na área específica (questão 2), a extensão da prova (questão 3), clareza e objetividade dos enunciados (questão 4), adequação das informações/instruções fornecidas para a resolução das questões (questão 5), confirmação da abordagem dos conteúdos durante o curso (questão 6), estudo dos conteúdos (questão 7) e avaliação do tempo ofertado e utilizado pelos estudantes na resolução das questões (questão 8).

A fim de descrever as variáveis, foram utilizados frequências e percentuais. Para correlacionar as variáveis ordinais entre si, foi empregado o coeficiente de correlação de Spearman e para correlacionar variáveis ordinais com variáveis categóricas (Questão 6), foi empregada a técnica multivariada, análise de correspondência. $\mathrm{O}$ nível de significância considerado foi $p<0,05$ e o software estatístico empregado foi o SPSS-15®.

\section{RESULTADOS}

Todos os 284 estudantes matriculados no $1^{\circ}$ semestre de 2014 na Faculdade de Odontologia da FCMS/JF participaram do TP 2014 e responderam o questionário de percepção. Destes, 215 (75,7\%) eram do gênero feminino e 69 (24,3\%) do gênero masculino.

Houve correlação significativa entre grau de dificuldade do teste com os períodos, sendo maior a dificuldade para os estudantes iniciantes e menor para os dos últimos períodos. Na questão 1 (conteúdos da área básica), observa-se maior percentual de escolha da opção "c - Médio" quanto mais avançados os períodos $\left(1^{\mathrm{o}} \mathrm{p}: 31,0 \%, 2^{\mathrm{o}} \mathrm{p}\right.$ : $50,0 \%, 3^{\circ} \mathrm{p}: 46,4 \%, 4^{\circ} \mathrm{p}: 64,5 \% .5^{\circ} \mathrm{p}: 79,5 \%, 6^{\circ} \mathrm{p}$ : $59,1 \%, 7^{\circ}$ p: $75,8 \%$ e $\left.8^{\circ} \mathrm{p}: 90,0 \%\right)$ e decréscimo da escolha pela opção "d- Difícil" ( $1^{\mathrm{o}} \mathrm{p}$ : $42,9 \%, 2^{\circ} \mathrm{p}$ : $34,0 \%, 3^{\circ}$ p: $33,9 \%, 4^{\circ}$ p: $25,8 \% .5^{\circ}$ p: $15,4 \%, 6^{\circ}$ p: $27,3 \%, 7^{\circ}$ p: $9,1 \%$ e $8^{\circ} \mathrm{p}: 0 \%$ ) (figura 1, correlação de Spearman $=-0,262 ; p=0,000)$.

$\mathrm{Na}$ questão 2 (componente específico) o mesmo pode ser observado (letra "c - Médio" - $1^{\circ}$ p: $19,0 \%, 2^{\circ}$ p: $56,0 \%, 3^{\circ}$ p: $57,1 \%, 4^{\circ}$ p: $67,7 \% .5^{\circ}$ p: $61,5 \%, 6^{\circ} \mathrm{p}: 77,3 \%, 7^{\circ} \mathrm{p}: 66,7 \%$ e $8^{\circ} \mathrm{p}: 100,0 \%$ e letra “d - Difícil" - $1^{\mathrm{o}} \mathrm{p}: 57,1 \%, 2^{\mathrm{o}} \mathrm{p}: 34,0 \%, 3^{\circ} \mathrm{p}$ : $33,9 \%, 4^{\circ} \mathrm{p}: 25,8 \% .5^{\circ} \mathrm{p}: 38,5 \%, 6^{\circ} \mathrm{p}: 18,2 \%, 7^{\circ} \mathrm{p}$ : $15,2 \%$ e $8^{\circ} \mathrm{p}: 0,0 \%$ ) (figura 2 , correlação de Spearman $=-0,259 ; p=0,000)$. As opções "a Muito fácil", "b - Fácil” e "e - Muito difícil” foram pouco marcadas, com média de 5 a $15 \%$ para as duas questões.

Quanto à percepção do estudante sobre a extensão da prova em relação aos períodos, observou-se que $177(62,5 \%)$ a consideraram adequada, $67(23,7 \%)$ longa, $36(12,7 \%)$ muito longa, $2(0,7 \%)$ curta, $1(0,3 \%)$ muito curta e 1 $(0,3 \%)$ não respondeu a esta pergunta (tabela 1$)$. Apesar de se observar tendência à percepção adequada da extensão da prova, não houve correlação significativa desta variável com os períodos (correlação de Spearman $=-0,104 ; p=$ $0,08)$. 


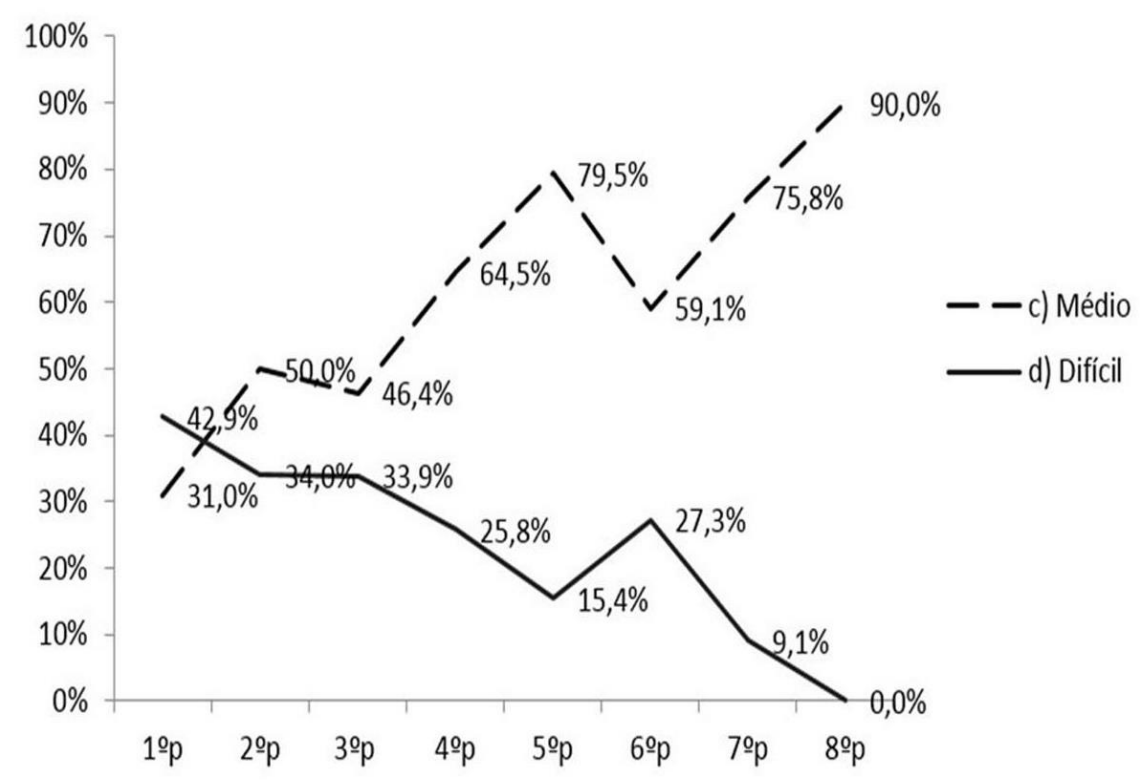

Figura 1. Correlação entre o grau de dificuldade do TP2014 na área básica com os períodos. Observase tendência ao decréscimo da opção "d - difícil" e aumento da opção "c - médio" quanto mais avançados no curso estão os estudantes (correlação de Spearman $=-0,262 ; p=0,000$ )

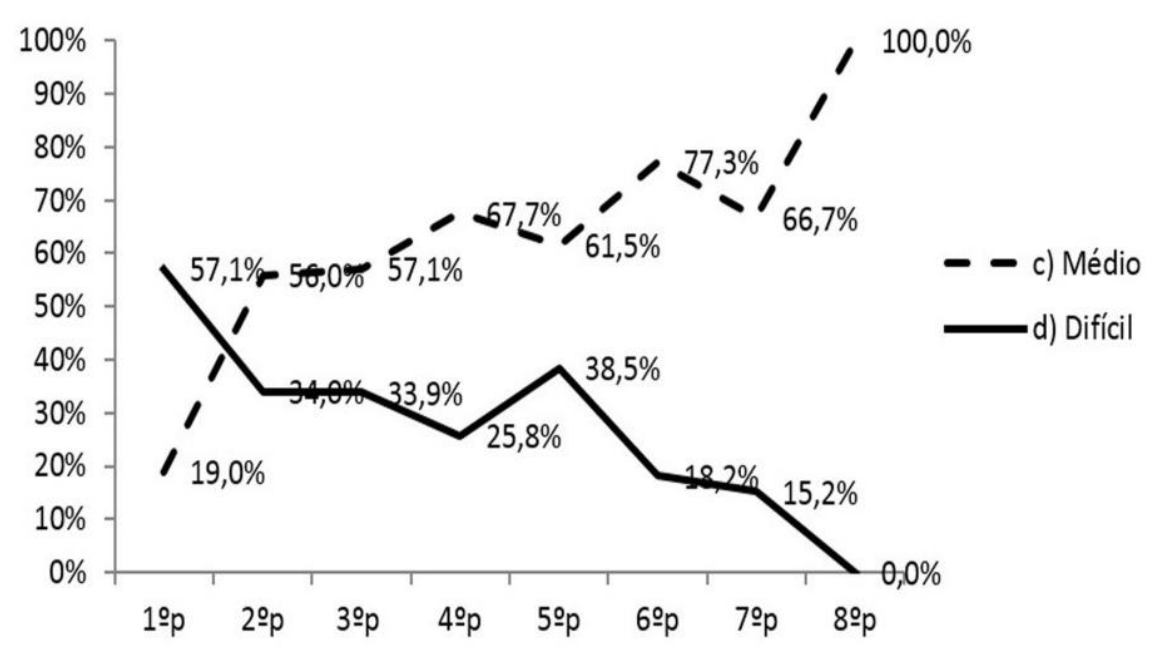

Figura 2. Correlação entre o grau de dificuldade do TP2014 na área específica com os períodos. Observa-se tendência ao decréscimo da opção "d - difícil" e aumento da opção "c - médio" quanto mais avançados no curso estão os estudantes (correlação de Spearman $=-0,259 ; p=0,000$ ) 
Tabela 1. Relação da extensão da prova com os períodos (correlação de Spearman $=-0,104 ; p=0,08$ )

\begin{tabular}{c|cccccc}
\hline \multirow{2}{*}{ Período } & & \multicolumn{5}{c}{ Extensão da prova } \\
& a) Muito longa & b) Longa & c) adequada & d) curta & e) muito curta & Total \\
\hline $1^{\mathbf{o}}$ & $2(4,8 \%)$ & $8(19,0 \%)$ & $32(76,2 \%)$ & 0 & 0 & 42 \\
$2^{\mathbf{o}}$ & $5(10,0 \%)$ & $12(24,0 \%)$ & $33(66,0 \%)$ & 0 & 0 & 50 \\
$3^{\mathbf{o}}$ & $7(12,5 \%)$ & $17(30,4 \%)$ & $31(55,45 \%)$ & 0 & $1(1,8 \%)$ & 56 \\
$4^{\mathbf{o}}$ & $3(9,7 \%)$ & $7(22,6 \%)$ & $20(64,5 \%)$ & $1(3,2 \%)$ & 0 & 31 \\
$5^{\mathbf{o}}$ & $8(20,5 \%)$ & $7(17,9 \%)$ & $24(61,5 \%)$ & 0 & 0 & 39 \\
$6^{\mathbf{o}}$ & $4(18,2 \%)$ & $3(13,6 \%)$ & $15(68,2 \%)$ & 0 & 0 & 22 \\
$7^{\mathbf{o}}$ & $6(18,2 \%)$ & $11(33,3 \%)$ & $15(45,5 \%)$ & $1(3,0 \%)$ & 0 & 33 \\
$8^{\mathbf{o}}$ & $1(10,0 \%)$ & $2(20,0 \%)$ & $7(70,0 \%)$ & 0 & 0 & 10 \\
Total & $36(12,7 \%)$ & $67(23,7 \%)$ & $177(62,5 \%)$ & $2(7,0 \%)$ & $1(4 \%)$ & 283 \\
\hline
\end{tabular}

Quando questionada a clareza e objetividade dos enunciados, constatou-se que a maioria dos estudantes assinalam mais as letras "a - Sim, todos" $(36,7 \%)\left(1^{\circ} \mathrm{p}: 45,2 \%, 2^{\circ} \mathrm{p}: 50,0 \%, 3^{\circ} \mathrm{p}: 35,7 \%, 4^{\circ} \mathrm{p}\right.$ : $45,2 \% .5^{\circ} \mathrm{p}: 25,6 \%, 6^{\circ} \mathrm{p}: 27,3 \%, 7^{\circ} \mathrm{p}: 24,2 \%$ e $8^{\circ} \mathrm{p}$ : $20,0 \%)$ e "b - Sim, a maioria" (50,5\%) (1'p: 50,0\%, $2^{\circ} \mathrm{p}: 40,0 \%, 3^{\circ} \mathrm{p}: 48,0 \%, 4^{\mathrm{o}} \mathrm{p}: 41,9 \% .5^{\circ} \mathrm{p}: 61,5 \%$, $6^{\circ} \mathrm{p}: 45,0 \%, 7^{\circ} \mathrm{p}: 63,6 \%$ e $\left.8^{\circ} \mathrm{p}: 70,0 \%\right)$.

Porém, na análise de correspondência das alternativas por período, observa-se menor percepção da clareza e objetividade pelos estudantes dos últimos períodos, de forma últimos períodos.

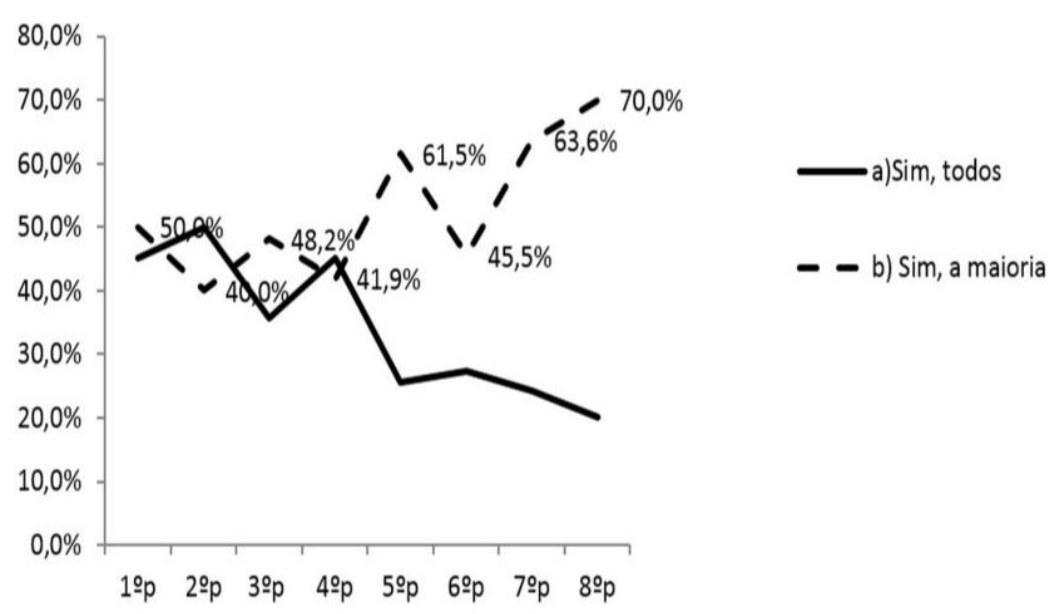

Figura 3. Correlação entre clareza e objetividade das questões da prova e os períodos. Do $1^{\circ}$ ao $4^{\circ}$ períodos observa-se que o distrator "b - sim a maioria", obteve entre 40 e $50 \%$, enquanto que nos últimos períodos ascende para até $70 \%$ 
Sobre a correlação da questão 5 (adequação das informações/instruções fornecidas para a resolução das questões) com os períodos, apesar da maioria dos estudantes responderem letra " $b$ - Sim, em todas elas"; com $41 \%$ de todas as alternativas ( $1^{\circ} \mathrm{p}: 42,9 \%, 2^{\circ} \mathrm{p}: 46,0 \%, 3^{\circ} \mathrm{p}: 35,7 \%, 4^{\circ} \mathrm{p}: 48,4 \%$, $5^{\circ}$ p: $35,9 \%, 6^{\circ}$ p: $50,0 \%, 7^{\circ}$ p: $33,3 \%$ e $8^{\circ}$ p: $\left.40,0 \%\right)$ e letra "c - Sim, na maioria delas", com $43 \%$ de todas as alternativas $\left(1^{\circ} \mathrm{p}: 35,7 \%, 2^{\circ} \mathrm{p}: 40,0 \%, 3^{\circ} \mathrm{p}\right.$ : $44,6 \%, 4^{\circ} \mathrm{p}: 29,0 \%, 5^{\circ} \mathrm{p}: 53,8 \%, 6^{\circ} \mathrm{p}: 45,5 \%, 7^{\circ} \mathrm{p}$ : $54,5 \%$ e $8^{\circ}$ p: 50,0\%); não houve correlação estatística significativa das alternativas com os períodos, como ilustrado na figura 4 (correlação de Spearman $=0,018 ; p=0,761)$.

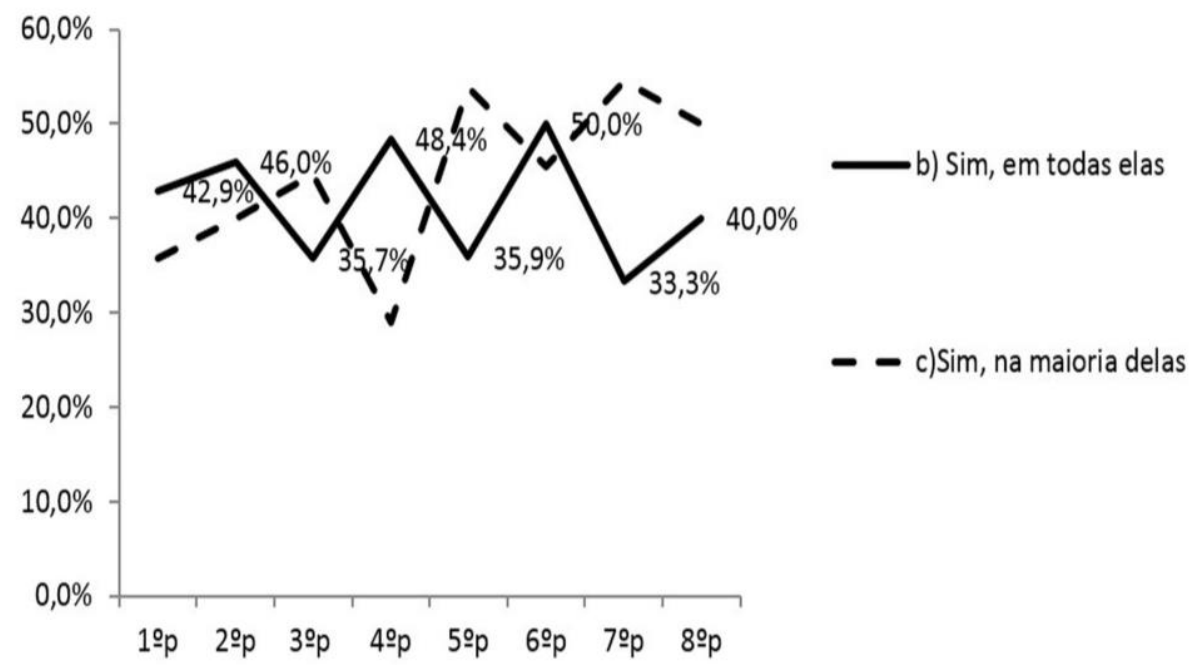

Figura 4. Correlação entre a percepção sobre as informações/instruções fornecidas para a resolução das questões do TP2014 com os períodos. Observa-se que a maioria dos estudantes responderam "b - sim, em todas elas" ou "c - sim, na maioria delas", com oscilações entre 30 a $55 \%$ (correlação de Spearman $=0,018 ; p=0,761$ )

Quando os acadêmicos foram questionados sobre a maior dificuldade encontrada para responder a prova (questão 6), após aplicação da técnica multivariada da análise de correspondência, percebe-se que, de forma significativa, os primeiros períodos (primeiro ao quinto) aproximam-se mais da letra "a - não estudou ainda a maioria desses conteúdos"; o sexto e sétimo aproximam-se mais das letras " $\mathrm{b}$ - forma diferente de abordagem do conteúdo" e "d - falta de motivação para fazer a prova", enquanto que o oitavo período apresenta-se com comportamento atípico, como ilustrado na figura 5 e tabela 2 (correlação de Spearman = 0,069, $p=0,000$ ). 


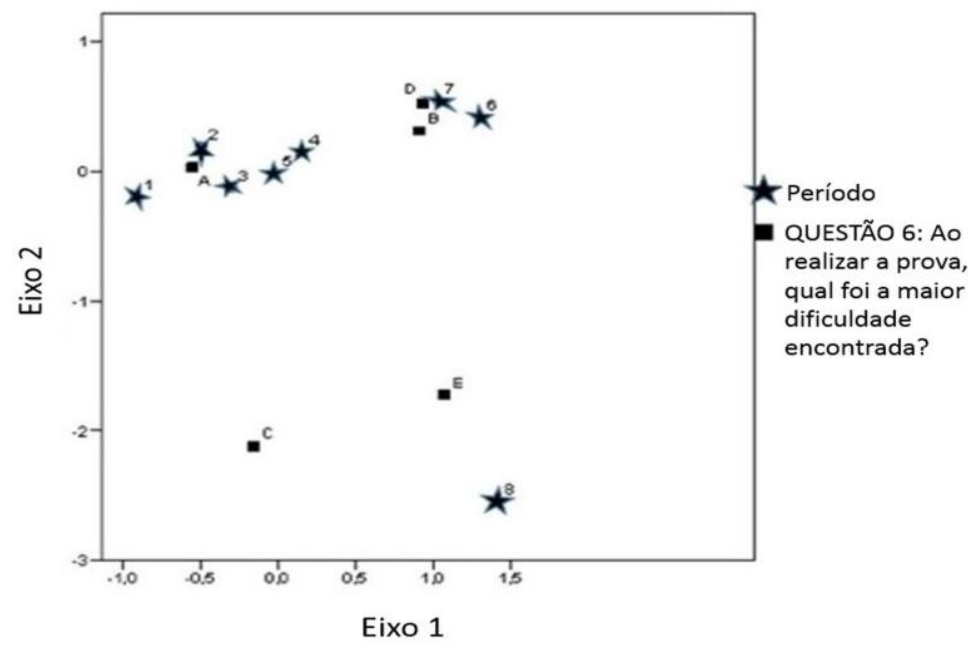

Figura 5. Correlação entre os distratores da questão 6 e os períodos. Percebe-se que do primeiro ao terceiro períodos há uma maior aproximação da opção "a - não estudou ainda a maioria desses conteúdos, sexto e sétimo aproximam-se da opção "d - falta de motivação para fazer a prova" e o oitavo período apresenta-se com comportamento atípico (correlação de Spearman $=0,069, p=0,000$ )

Tabela 2. Respostas à questão 6 quanto à maior dificuldade encontrada pera responder a prova entre os períodos (correlação de Spearman $=0,069, p=0,000$ )

\begin{tabular}{c|cccccc}
\hline Período & $\begin{array}{c}\text { a) Não estudou } \\
\text { ainda a maioria } \\
\text { desses conteúdos }\end{array}$ & $\begin{array}{c}\text { b) Forma } \\
\text { diferente de } \\
\text { abordagem do } \\
\text { conteúdo }\end{array}$ & $\begin{array}{c}\text { c) Espaço } \\
\text { insuficiente para } \\
\text { responder às } \\
\text { questões }\end{array}$ & $\begin{array}{c}\text { d) Falta de } \\
\text { motivação } \\
\text { para fazer a } \\
\text { prova }\end{array}$ & $\begin{array}{c}\text { e) Não tive } \\
\text { qualquer tipo de } \\
\text { dificuldade para } \\
\text { responder a prova }\end{array}$ & Total \\
\hline $1^{\mathbf{o}}$ & $39(22,9 \%)$ & $1(1,7 \%)$ & $1(20 \%)$ & $0(0 \%)$ & $1(6,3 \%)$ & 42 \\
$2^{\mathbf{o}}$ & $40(23,0 \%)$ & $6(10,3 \%)$ & $0(0 \%)$ & $3(10,3 \%)$ & $1(6,3 \%)$ & 50 \\
$3^{\mathbf{o}}$ & $38(21,8 \%)$ & $9(15,5 \%)$ & $3(60,0 \%)$ & $4(13,8 \%)$ & $1(6,3 \%)$ & 55 \\
$4^{\mathbf{o}}$ & $18(10,3 \%)$ & $7(12,1 \%)$ & $0(0 \%)$ & $4(13,8 \%)$ & $2(12,5 \%)$ & 31 \\
$5^{\mathbf{o}}$ & $25(14,4 \%)$ & $7(12,1 \%)$ & $0(0 \%)$ & $4(13,8 \%)$ & $3(18,8 \%)$ & 39 \\
$6^{\mathbf{o}}$ & $4(2,3 \%)$ & $11(19,0 \%)$ & $0(0 \%)$ & $5(17,2 \%)$ & $2(12,5 \%)$ & 22 \\
$7^{\mathbf{o}}$ & $9(5,2 \%)$ & $14(24,1 \%)$ & $0(0 \%)$ & $8(27,6 \%)$ & $2(12,5 \%)$ & 33 \\
$8^{\mathbf{o}}$ & $1(6 \%)$ & $3(5,2 \%)$ & $1(20 \%)$ & $1(3,4 \%)$ & $4(25 \%)$ & 10 \\
Total & 178 & 58 & 5 & 29 & 16 & 282 \\
\hline
\end{tabular}

Sobre a correlação do estudo dos conteúdos com os períodos, a percepção dos acadêmicos de Odontologia é de que há uma percepção significativa de ganho cognitivo com o decorrer dos períodos, expressos pela tendência a aumentar a marcação da letra "d" estudou e aprendeu muitos desses conteúdos $\left(1^{\circ} \mathrm{p}: 4,8 \%, 2^{\circ} \mathrm{p}: 4,0 \%, 3^{\circ} \mathrm{p}\right.$ :
$16,1 \%, 4^{\circ} \mathrm{p}: 38,7 \% .5^{\circ} \mathrm{p}: 33,3 \%, 6^{\circ} \mathrm{p}: 72,7 \%, 7^{\circ} \mathrm{p}:$ $60,6 \%$ e $8^{\circ}$ p: $70,0 \%$ ) e diminuir a opção pela letra "a" não estudou ainda a maioria desses conteúdos (1 ${ }^{\circ}$ p: $95,2 \%, 2^{\circ}$ p: $88,0 \%, 3^{\circ}$ p: $57,1 \%, 4^{\circ}$ p: $29,0 \%$. $5^{\circ}$ p: $25,6 \%, 6^{\circ}$ p: $13,6 \%, 7^{\circ}$ p: $6,1 \%$ e $8^{\circ}$ p: $\left.0,0 \%\right)$, como ilustrado na figura 6 (correlação de Spearman $=0,651 ; p=0,000)$. 


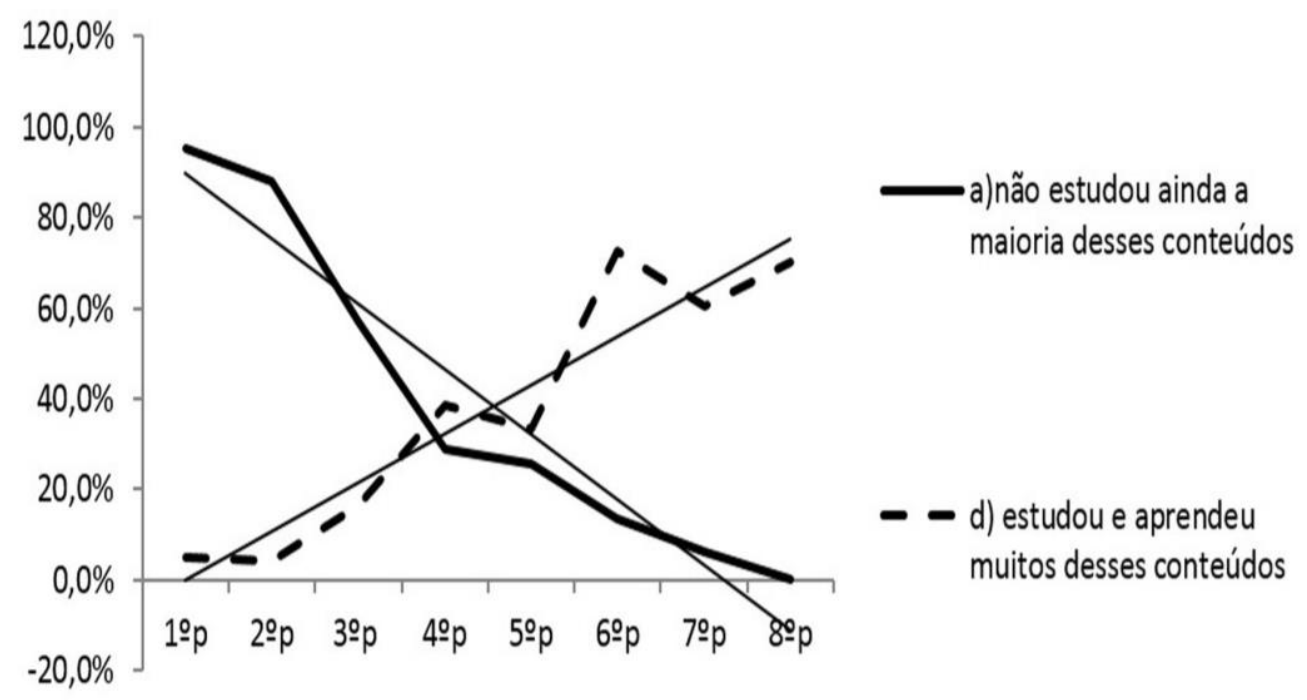

Figura 6. Correlação entre os distratores da questão 7 e os períodos. Percebe-se uma tendência a marcar mais a opção "d - estudou e aprendeu muitos desses conteúdos" e menos a opção "a - não estudou ainda a maioria desses conteúdos" conforme o estudante avança no curso (correlação de Spearman $=0,651 ; p=0,000)$

Já em relação ao tempo ofertado e utilizado pelo acadêmico para concluir o TP 2014, 67,4\% dos estudantes marcaram a opção entre uma e duas horas, $25,8 \%$ marcaram entre duas e três horas, $4 \%$ não conseguiram terminar a prova,
$3,9 \%$ fizeram em menos de uma hora e $2,5 \%$ realizaram entre três e quatro horas, não havendo correlação significativa com os períodos, como demonstrado na tabela 3 (correlação de Spearman $=0,054 ; p=0,371)$.

Tabela 3. Avaliação do tempo ofertado e utilizado pelos estudantes para a resolução das questões de acordo com os períodos (correlação de Spearman $=0,054 ; p=0,371$ )

\begin{tabular}{ccccccc}
\hline Período & $\begin{array}{c}\text { a) Menos de } \\
\text { uma hora }\end{array}$ & $\begin{array}{c}\text { b) Entre uma } \\
\text { e duas horas }\end{array}$ & $\begin{array}{c}\text { c) Entre } \\
\text { duas e três } \\
\text { horas }\end{array}$ & $\begin{array}{c}\text { d) Entre três } \\
\text { e quatro } \\
\text { horas }\end{array}$ & $\begin{array}{c}\text { e) Quatro } \\
\text { horas e não } \\
\text { consegui } \\
\text { terminar }\end{array}$ & Total \\
\hline $1^{\mathbf{o}}$ & $3(7,3 \%)$ & $32(78,0 \%)$ & $5(12,02 \%)$ & 0 & $1(2,4 \%)$ & 41 \\
$2^{\mathbf{o}}$ & $4(8,0 \%)$ & $30(60,0 \%)$ & $15(30,0 \%)$ & $1(2,0 \%)$ & $0(0 \%)$ & 50 \\
$3^{\text {o }}$ & $3(5,5 \%)$ & $30(54,5 \%)$ & $20(36,4 \%)$ & $2(3,6 \%)$ & $0(0 \%)$ & 55 \\
$4^{\mathbf{o}}$ & 0 & $21(67,7 \%)$ & $9(29,0 \%)$ & $1(3,2 \%)$ & $0(0 \%)$ & 31 \\
$5^{\mathbf{o}}$ & 0 & $27(71,1 \%)$ & $10(26,3 \%)$ & $1(2,6 \%)$ & $0(0 \%)$ & 38 \\
$6^{\mathbf{o}}$ & 0 & $17(77,3 \%)$ & $5(22,7 \%)$ & 0 & $0(0 \%)$ & 22 \\
$7^{\mathbf{o}}$ & $1(3,0 \%)$ & $25(75,8 \%)$ & $6(18,2 \%)$ & $1(3,0 \%)$ & $0(0 \%)$ & 33 \\
$8^{\mathbf{o}}$ & 0 & $6(66,7 \%)$ & $2(22,2 \%)$ & $1(11,1 \%)$ & $0(0 \%)$ & 9 \\
Total & $11(3,9 \%)$ & $188(67,4 \%)$ & $72(25,8 \%)$ & $7(2,5 \%)$ & $1(4 \%)$ & 279 \\
\hline
\end{tabular}




\section{DISCUSSÃO}

A elaboração do teste de progresso é um desafio $^{10,11}$. Para tanto, é necessário que o realizador do teste tenha um objetivo claro, com o propósito de produzir informações como: o nível médio de conhecimento para um grupo de acadêmicos e individualmente, tanto em um ponto específico do curso, como ao longo deste; a possibilidade de comparar instituições que realizam o mesmo teste e a autoavaliação institucional, permitindo a análise da relação entre conteúdo e estrutura curricular da graduação ${ }^{4,11}$.

Este estudo possibilitou uma avaliação da percepção dos acadêmicos com relação ao grau de dificuldade da prova, sua extensão, clareza e objetividade dos enunciados, adequação das informações/instruções fornecidas para a resolução das questões, confirmação da abordagem dos conteúdos durante o curso, estudo dos conteúdos pelos acadêmicos e avaliação do tempo ofertado e utilizado na resolução das questões. Assim, observou-se que a prova foi considerada mais difícil, tanto na área básica quanto na específica, quanto menos avançados os estudantes estão no curso e, com o passar do tempo, em períodos mais avançados esta dificuldade diminui para ambas as áreas. Este resultado é observado em outras instituições, que utilizam o teste de progresso de maneira formativa em suas matrizes curriculares. Assim, quando se analisa a porcentagem de acertos dos alunos na UEL, percebe-se aumento no ganho cognitivo com o decorrer do curso ${ }^{4}$. Outros estudos, como o realizado na King Saud bin Abdulaziz University for Health Sciences (KSAUHS) ${ }^{12}$ e na Faculdade de Medicina na Universidade de São Paulo (USP) ${ }^{13}$, corroboram a afirmação de que os acadêmicos percebem melhora da aquisição do conhecimento através do teste de progresso.

Ao analisar a extensão da prova neste estudo, alguns acadêmicos a consideraram longa ou muito longa, entretanto a maioria considerou adequada. Muitas universidades realizam o teste com 100 questões ou mais como as universidades inglesas e americanas, que utilizam entre 100 a 125; holandesas com 200 e mesmo no Brasil há uma variação entre 120 a $150^{2,4,10,14,15}$. A qualidade das questões deve ser considerada para a definição do número a ser abordado no teste. Quando a qualidade é superior, não justifica o aumento de questões, pois o número excessivo pode resultar no cansaço dos acadêmicos, prejudicando o seu desempenho ${ }^{4}$.

Quanto à clareza e objetividade dos enunciados da prova, foi observado que apesar da diferença de conhecimento de cada período, a maioria dos estudantes considerou a prova objetiva e clara, entretanto essa percepção diminuiu significativamente nos últimos períodos. Em relação à adequação das informações/instruções fornecidas para a resolução das questões, em torno de $84 \%$ dos estudantes (somando-se as opções b e c) percebem que as informações e instruções fornecidas estão adequadas para a resolução das questões, sem significância estatística observada entre os períodos. Levando em consideração que cada instituição tem seus próprios métodos de formulação da prova, algumas diferenças, tais como questões de verdadeiro ou falso ou questões discursivas, podem interferir na interpretação do acadêmico, sendo de extrema importância que qualquer sistema de avaliação inclua medidas confiáveis e precisas de aquisição de conhecimento $^{4,16}$.

Quando se trata da abordagem dos conteúdos durante o curso, nos períodos iniciais o desconhecimento do conteúdo foi a alternativa mais marcada, o que já era esperado na formulação de hipóteses para este trabalho, já que o objetivo do teste de progresso é que o acadêmico obtenha um ganho cognitivo com o decorrer do curso. Este resultado é corroborado por diversos estudos que reforçam o objetivo do teste de progresso que é o progresso longitudinal do processo ensinoaprendizagem $^{1-3,6,17}$. Um estudo realizado na 
Ludwig-Maximilians-Universität (LUM) em Munique procurou avaliar não só o crescimento cognitivo do acadêmico, mas a aquisição do conhecimento permanente e concluiu que a introdução do teste de progresso pode contribuir para a permanência do conhecimento; demonstrando que o aumento deste ocorre continuamente e depende das experiências dos acadêmicos tanto na parte clínica quanto nos seus estudos teóricos ${ }^{18}$. Já os estudantes dos períodos finais, relataram falta de motivação para fazer a prova. Estudos demonstram que um dos fatores que influenciam os resultados do TP foi exatamente a desmotivação dos acadêmicos ao realizá-lo e que a simples imersão na prática não é suficiente para desenvolver competências e resolver um problema específico $^{4,19}$. É necessário organizar o processo de ensino-aprendizagem, estimulando o desenvolvimento de competência profissional, com ênfase nas habilidades psicomotoras e cognitivas. Para isso, estabelecer avaliações formativas, cujo foco não seja meramente classificatório, que promovam aprendizagem contínua, torna-se relevante $^{19}$.

Em várias universidades o $\mathrm{TP}$ possui em média de 100 a 200 questões e o tempo de realização entre duas horas e meia a três horas $2,10,14,15$. Planejou-se um tempo total de quatro horas para o acadêmico concluir o TP2014, com 100 questões. Dos 284 alunos que o realizaram, 177 consideraram o tempo adequado, apesar de $62,3 \%$ dos acadêmicos terem realizado a prova com apenas uma a duas horas e $23,7 \%$ deles, em duas a três horas. Este resultado sugere que, assim como acontece em países desenvolvidos, o teste pode ser, de fato, planejado com menos tempo de duração.

As fortalezas do nosso estudo referem-se ao número de acadêmicos que participaram da pesquisa e o fato do estudo proporcionar o diagnóstico por parte dos professores das disciplinas avaliadas quanto à avaliação do estudante em relação ao conteúdo abordado. No entanto, uma grande limitação da realização do TP é a falta de motivação dos acadêmicos dos períodos mais avançados, uma vez que o teste não tem caráter somativo na instituição. Esse fato influencia o estudo, porque desmotiva, também, o preenchimento adequado da avaliação. Outra limitação foi a realização do estudo em apenas um momento. Outros estudos estão sendo realizados para que possa haver uma comparação longitudinal entre testes realizados em tempos diferentes.

Segundo as Diretrizes Curriculares Nacionais (DCN) para os cursos de graduação em Odontologia, instituídas pela Resolução CNE/CES 3, de 19 de fevereiro de 2002, deverão ser utilizadas metodologias e critérios para acompanhamento e avaliação do processo ensino-aprendizagem e do próprio curso, em consonância com o sistema de avaliação e a dinâmica curricular definidos pela instituição de ensino superior à qual pertence ${ }^{20}$. Além disto, as DCN preconizam a aquisição de competências e habilidades gerais como atenção à saúde, tomada de decisões, comunicação, liderança, administração e gerenciamento bem como educação permanente. Neste estudo, pode-se observar que a falta de motivação dos estudantes dos últimos períodos para realizar o teste demonstra uma lacuna no desenvolvimento do espírito crítico ou falta de entendimento do processo, o que dificulta a tomada de decisão. Entretanto, perceber que há um ganho cognitivo dos conteúdos na relação TP com os períodos, atende a habilidade de educação permanente, onde os estudantes aprendem a aprender e passam a ter responsabilidade e compromisso com a sua educação, tornando-os futuros profissionais com capacidade de administrar e gerenciar sua profissão e então liderar com responsabilidade.

Sendo assim, o TP pode ser considerado uma forma de avaliação longitudinal do desenvolvimento cognitivo dos acadêmicos aplicado durante o curso de graduação e tem por 
objetivo não só avaliar o desempenho cognitivo dos acadêmicos, assim como aspectos do próprio curso de graduação, sem caráter de aprovação, reprovação ou classificação. Logo, a avaliação da percepção dos acadêmicos de Odontologia em relação ao TP contribui também para a construção de habilidades preconizadas pelas DCN, fornecendo informações confiáveis para o aprimoramento desta avaliação.

\section{CONCLUSÃO}

A percepção dos acadêmicos em relação ao TP demonstra que o mesmo está sendo realizado de forma adequada. Observou-se ainda que os períodos iniciais relataram desconhecimento do conteúdo, o que corrobora com o objetivo do teste, que é avaliar o ganho cognitivo dos acadêmicos à medida que avançam no curso.

\section{ABSTRACT \\ Perception of Dental students with regard to Progress Tests}

This study aimed to analyze the perception of students of the Dentistry of Progress Test (TP) 2014. Therefore, after completion of the TP, 284 students received a questionnaire, which addressed the perception of the degree of difficulty of the test in the basic and specific areas; the extent of the test; clarity and objectivity of statements; adequacy of the information / instructions provided to resolve the issues; confirmation of the content approach during the course; study of the contents by the students and evaluation of the time offered and used by the students to solve the questions. Spearman's correlation coefficient and correspondence analysis (SPSS-15 (C) were used. Thus, it was observed that the degree of difficulty of the test in the basic area and in the specific area was higher for the students of the first periods. With regard to the students' perception of extent of the test, the majority considered this, as well as the clarity and objectivity of the test to be adequate. Regarding the difficulty in performing the test, the students in the first periods reported ignorance of all content; and for the last periods, they reported a lack of motivation. Based on the results obtained, it could be concluded that the students' perception of the test demonstrated that it was being performed properly. Moreover, students in the initial periods were observed to report a lack of knowledge about the content, thus corroborating the objective of the test, which is to evaluate the cognitive gain of students as they progressed in the course.

Descriptors: Evaluation. Taxonomy. Professional Qualification. Education, Dental.

\section{REFERÊNCIAS}

1. Schuwirth LWT, Vleuten CPM. The use of progress testing. Perspect Med Educ. 2012 Mar; 1(1):24-30.

2. Ali K, Coombes L, Kay E, Tredwin C, Jones $\mathrm{G}$, Ricketts $\mathrm{C}$, et al. Progress testing in undergraduate dental education: the Peninsula experience and future opportunities. Eur J Dent Educ. 2016 Aug;20(3):129-34.

3. Sakai MH, Ferreira Filho OF, Matsuo T. Avaliação do crescimento cognitivo do estudante de medicina: aplicação do teste equalização no teste de progresso. Rev Bras Educ Med. 2011; 493(4):493-501.

4. Sakai MH, Ferreira Filho OF, Almeida MJ, Mashima DA, Marchese MC. Teste de Progresso avaliação do curso: dez anos de experiência da medicina da Universidade Estadual de Londrina. Rev Bras Educ Med. 2008;32(2):254-63.

5. Bennett J, Freeman A, Coombes L, Kay L, Ricketts C. Adaptation of medical progress testing to a dental setting. Med Teach. 2010;32(6):500-2.

6. Ali K, Zahra D, Tredwin C, Mcilwaine C, Jones G. Use of Progress Testing in a UK Dental Therapy and Hygiene Educational Program. J Dent Educ. 2018 Feb;82(2):1306. 
7. Goldwasser R, Fonseca V, Lobo MS, Coelho A, Santos EG, Pereira SMP. Seleção para a residência médica da Universidade Federal do RJ percepção dos candidatos sobre o modelo da prova. Rev Bras Educ Med. 2009;33(1):115-21.

8. Barreira C, Bidarra G, Monteiro F, VazRebelo P, Alferes Barreira V. Avaliação das aprendizagens no ensino superior. Percepções de professores e estudantes nas universidades portuguesas. Rev Ibero Am Educ Super. 2017;8(21):24-36.

9. Davok DF. Modelo de meta avaliação de processos de avaliação da qualidade de cursos de graduação.2006, 272f. [Tese]. Doutorado em Engenharia de Produção, UFSC, Florianópolis, SC.

10. Wade L, Harrison C, Hollands J, Mattick K, Ricketts C, Wass V. Student perceptions of the progress test in two settings and the implications for test deployment. Adv Health Sci Educ Theory Pract. 2012 Oct;17(4):573-83.

11. Ricketts C, Freeman A, Pagliuca G, Coombes L, Archer J. Difficult decisions for progress testing: how much and how often? Med Teach. 2010;32(6):513-5.

12. Al Alwan I, Al-Moamary M, Al-Attas N, Al Kushi A, AlBanyan E, Zamakhshary M, et al. The progress test as a diagnostic tool for a new PBL curriculum. Educ Health. 2011 Dec;24(3):493.

13. Tomic ER, Martins MA, Lotufo PA, Benseñor IM. Progress testing: Evaluation of four years of application in the school of medicine, University of São Paulo. Clinics. 2005;60(5):389-96.

14. Cecilio-Fernandes D, Kerdijkb W, Jaarsmaa ADDC, Tioa RA. Development of cognitive processing and judgments of knowledge in medical students: Analysis of progress test results. Med Teach. 2016 Nov;38(11):11259.

15. Coelho C, Zahra D, Ali K, Tredwin C. To accept or decline academic remediation: What difference does it make? Med Teach. 2019 July; 41(7):824-9.

16. Portanova R, Adelman M, Jollick JD, Schulers S, Modrzakowskim M, Soper E, et al. Student assessment in the Ohio University College of Osteopathic Medicine CORE system: progress testing and objective structured clinical examinations. Med Educ. 2000 Nov;100(10):707-12.

17. Pugh D, Regehr G. Taking the sting out of assessment: is there a role for progress testing? Med Educ. 2016 Jul;50(7):721-9.

18. Schmidmaier R, Holzer M, Angstwurm M, Nouns Z, Reincke M, Fischer MR. Querschnitt evaluation des Medizinischen Curriculums München (MeCuM) mitHilfe des Progress Tests Medizin (PTM). Humanmedizin. 2010;27(5) :1-14.

19. Dijksterhuis MGK, Scheele F, Schuwirth LWT, Essed GGM, Nijhuis JG, Braat DDM. Progress testing in postgraduate medical education. Med Teach. 2009 Oct;31(10):e464-8

20. Brasil. Ministério da Educação. Conselho Nacional de Educação. Câmara de Educação Superior. Resolução CNE/CES, de 19 de fevereiro e 2002 [Institui Diretrizes Curriculares Nacionais do Curso de Graduação em Odontologia]. Diário Oficial da República Federativa do Brasil. 2002, 04 de mar; Seção 1:10.

\section{Correspondência para:}

Fabiana Aparecida Mayrink de Oliveira e-mail: fabsmay@ hotmail.com Avenida Barão do Rio Branco, 1871/1105 6013-020 Juiz de Fora/MG 